\title{
Ein Vermächtnis oder «Alles hat seine Zeit»
}

\author{
Heinz Bhend ${ }^{a}$, Gerhard Schilling ${ }^{b}$ \\ ${ }^{a}$ Heinz Bhend, Facharzt Allgemeine Innere Medizin und Informatiker, Aarburg; ${ }^{b}$ Gerhard Schilling, pens. Hausarzt, ehem. Kommission Informatics/ \\ eHealth mfe, Stein am Rhein
}

Nach jahrelangem Engagement im Bereich Praxisinformatik und eHealth ziehen wir uns (von der IT-Bühne) zurück. Wir konnten erreichen, dass das Thema «Praxisinformatik» in der FMH angekommen ist und mit einer neuen Crew praxisrelevante Projekte angepackt werden. Wir waren nicht immer einfach, teilweise zu undiplomatisch, vielleicht auch zu forsch, oft wohl einfach zu früh. Uns ging es aber immer um die Sache an sich. Die hier angesprochenen Themen sind eine Art Checkliste der - nach unserer Ansicht - relevanten Fragen rund um Arztpraxis und eHealth. Es ist nun an den neuen Kräften, die Prioritäten richtig zu setzen, Bereiche wegzulassen oder andere aufzunehmen.

\section{Die Akteure rund um eHealth}

Im Bereich eHealth sind die niedergelassenen Ärztinnen und Ärzte (im Folgenden nur noch Ärzte genannt) die Hauptakteure und stehen damit zwangsläufig im Fokus. Die «Hausarzt-Gilde» stellt zahlenmässig den grössten Anteil und hat wohl die komplexesten Praxissettings, so dass diese Ärztegruppe federführend sein sollte oder zumindest nicht übergangen werden kann.

Seit über 20 Jahren besteht seitens der Ärzte (als Anwender) eine initial lockere und zunehmend intensivere Bindung zu dem eigenen Softwarelieferanten. Im Rahmen der gesteigerten Komplexität (praxisinternes Computernetz, Internet, Sicherheit, Backup usw.) wurde aus der Bindung eine eindimensionale Abhängigkeit. Die heterogene Gruppe der Softwareanbieter ist im VSFM (Verein Schweizer Fachhäuser für Medizininformatik) relativ locker assoziiert. - Bis vor kurzem prägten Ein-

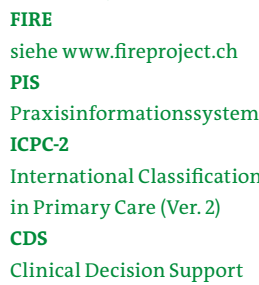

zelpraxen mit wenigen Arbeitsplätzen die Landschaft. Zunehmend sind Gruppenpraxen mit 10, 15, 20 und mehr Arbeitsplätzen im Vormarsch. Diese Entwicklung führte dazu, dass IT-Dienstleister im Bereich Hardware/ Vernetzung/Backup/Sicherheit die Bereitstellung der Informatik-Infrastruktur übernehmen und die traditionellen Anbieter von Praxisinformationssystemen (PIS) sich auf ihr Kerngeschäft zurückziehen.

Mit zunehmender Digitalisierung und Vernetzung bieten neue Akteure ihre Dienste an: Intermediäre kämpfen um den potentiell lukrativen Markt des Datenaustausches. Aktuell liefern sich Swisscom und Post ein Kopf-an-Kopf-Rennen mit je eigenen, teilweise untereinander noch inkompatiblen Systemen.

Seit zehn Jahren hat eHealth Suisse den Auftrag, ein Patientendossier (EPD = elektronisches Patientendossier) zu realisieren. Nach den fehlenden Realisierungschancen für eine zentrale Datenbank hat man sich für ein dezentrales Dossier entschieden, dessen Management (Zugriffsrechte, Zertifizierung, Verteilung auf diverse Stammgemeinschaften, unterschiedlicher Entwicklungsstand von Teilprojekten usw.) an Komplexität praktisch nicht mehr zu überbieten ist. Die Spitäler und Heime sind ab 2020 rsp. 2022 verpflichtet, ein elektronisches Patientendossier anzubieten. Für Bürger und niedergelassene Ärzte bleibt es freiwillig.

Die folgende Auflistung von Themen, die Formulierung von Thesen und möglichen Konsequenzen sollen die Diskussion über eHealth anregen, um ein Abenteuer möglichst zu vermeiden. 
Insofern ist es keine wissenschaftliche Abhandlung, sondern gibt die Meinung der Autoren wieder, im Bewusstsein, dass diese nicht die einzige und nicht sakrosankt ist.

\section{Herausforderungen und Konsequenzen}

- Herausforderung: Das EPD schafft ein verändertes Umfeld: Seitens der Ärzteschaft werden zusätzlicher Zeitaufwand und negative finanzielle Konsequenzen befürchtet. Die Möglichkeit, dass sich schleichend eine ablehnende Grundhaltung ausbreitet, ist real. Dies später korrigieren zu wollen ist unrealistisch. Die niedergelassenen Ärzte warten ab und lassen sich allenfalls durch Voten der Verbände motivieren. Konsequenz: Bis Mitte 2018 muss klar sein, ob die Ärzteschaft mitmacht oder nicht. Beides, sowohl abseitsstehen als auch aktiv mitgestalten, muss begründet und nachvollziehbar sein und darf weder zu einer finanziellen noch zeitlichen Mehrbelastung führen.

- eHealth Suisse: Die Vernehmlassungen der eHealthStrategie von Bund und Kantonen geschieht mehrheitlich ohne substantiellen Miteinbezug der (dereinst direkt betroffenen) Ärzteschaft. Bisher konnten Stellungnahmen im Rahmen von Vernehmlassungen eingebracht werden. Im Hinblick auf die Realisierung haben erste Treffen zwischen eHealth Suisse und den Softwarelieferanten stattgefunden, wiederum ohne Einbezug der niedergelassenen Ärzte. Sich nur im Rahmen von Vernehmlassungen einbringen zu können reicht in der Umsetzungsphase nicht mehr aus. Deshalb: Keine weiteren eHealth-Schritte mehr ohne direkten repräsentativen Miteinbezug der Ärzteschaft.

- Datenaustausch: Prioritär ist der Datenaustausch von (Software-)System A nach (Software-)System B. Dass dies möglich ist, hat z.B. SMEEX schon vor Jahren bewiesen. Die Softwarefirmen haben ihr Versprechen der Implementierung nicht gehalten, die Ärzte haben diese zu wenig konsequent gefordert. Der Datenaustausch eines einzelnen (Patienten-)Datensatzes (z.B. Arztwechsel) als auch aller Patientendaten (z.B. Anbieterwechsel) muss zeitnah realisiert werden.

- Lead/Clearingstelle: Verschiedene Akteure tragen ihre Wünsche für Integrationen, Ergänzungen und Schnittstellen an die Softwarefirmen heran. FIRE möchte den Exporter optimieren, MC-Organisationen haben eigene Monitore entwickelt für «Chronic Care» usw. Die Wünsche an die Tools betreffend Datenaustausch und Schnittstellen werden in den nächsten Jahren exponentiell ansteigen. Falls weiterhin unkoordiniert gefordert und entwickelt wird, steigert das Komplexität und Kosten.
Für Dritte darf es nur einen Ansprechpartner geben im Bereich eHealth/Praxisinformatik. Sowohl seitens ärztlicher Institutionen/Fachgruppen (mfe, FMH, KHM, IHAM, MC-Organisationen usw.) als auch seitens der Industrie/Behörden/Instanzen (VSFM, BAG, eHealth Suisse, Intermediäre, Kantone) müssen die Wünsche/ Anforderungen über eine Clearingstelle laufen.

- Kommunikation: Schnittstellen sind der Ausgangspunkt. Von eHealth wird gemeinhin Effizienz- und Qualitätssteigerung erwartet. Als unabdingbare Voraussetzung dafür müssen (valide, strukturierte) Daten exportierbar und mit tiefer Integration in die PIS importierbar sein.

Ein strukturierter bidirektionaler Datenaustausch ist für Diagnoselisten (ICD-10) und Medikamentenlisten (GTIN) anzustreben. Praxisintern werden ICD-10-Diagnosen möglichst auf ICPC-Codes "gemappt».

- Doppelte Erfassung: Infolge fehlender Integration starten gewisse Projekte mit separaten Erfassungsmasken für Daten, die in den PIS schon vorhanden sind. Dies ist kontraproduktiv in Bezug auf Effizienz und damit für die Akzeptanz der elektronischen Datenverarbeitung.

Jeder Ansatz von doppelter Dateneingabe wird konsequent abgelehnt.

- Elektronische Dokumentation: Der Erfolg von eHealth hängt u.a. auch von einer kritischen Masse von Anwendern ab. Nur elektronisch dokumentierende Ärzte können potentiell ein EPD managen. Solange die elektronische Dokumentation keinen direkten praxisinternen Mehrwert bietet, wird der grosse Durchbruch nicht kommen. Dass elektronisch dokumentieren "modern» ist, reicht mittelfristig nicht aus.

Die Verbände lancieren eine neue Initiative mit dem Ziel «2025 sind alle angekommen». Dieses Ziel ist klar zu avisieren, Mittel sind bereitzustellen für Information, Support, Beratung, Motivation usw.

- Datenerfassung: Je besser die primär erfassten Daten sind, desto grösser ist der Nutzen für nachgelagerte (praxis-)interne und externe Prozesse. Längst gibt es Möglichkeiten der IT-Unterstützung für eine vollständigere Erfassung qualitativ besserer Daten.

Im Rahmen der Überarbeitung der eKG-Prozesse werden Tools zur optimierten Dateneingabe definiert und entsprechend eingefordert (z.B. Thesaurus).

- Datenqualität: Die aktuelle Qualität der in den Praxen gespeicherten Daten ist ungenügend. Erst wenn die Daten praxisintern wiederverwendet werden und damit "Korrektur-Algorithmen» etabliert sind, steigert sich die Qualität der Daten.

Erweiterung der PIS durch Tools mit direktem praxisinternem Mehrwert (z.B. klinische Relationen). Vgl. Schweiz Ärzteztg. 2015;96(43):1579-81. 
- Ärzteeigener Datenexport: Aktuell werden unterschiedliche Daten (z.B. FIRE, TrustX) mit unterschiedlichen Zielen aus den PIS exportiert. Grundsätzlich sollten alle Daten aus den PIS anonymisiert und oder pseudonymisiert exportierbar sein.

Export von klinischen Parametern (z.B. FIRE) und Transfer von Kostendaten (Trust-Center) sollten neu aufgegleist und vereinheitlicht werden. Ein vollständiger Export (inklusive Freitextfeldern) aller Daten in ein ärzteeigenes (Trust-)Data-Warehouse müsste längst angepackt werden. Von diesem aus können dann gezielt Daten zur Verfügung gestellt werden.

- State oft the Art der elektronischen Dokumentation: Aktuell spiegelt die Heterogenität der Dokumentationsart die individuelle Vielfalt der Ärzte ab. Je individueller die Wünsche sind, desto teurer und komplexer sind die Systeme und umso schwieriger ist der standardisierte Export im Sinne einer effizienten Kommunikation.

Ein minimales Datenset sowie eine Minimalvariante der elektronischen Dokumentation sollten "verpflichtend» für jede Konsultation sein (im Sinne von "good practice»).

- Medikamentenmanagement: Die PIS bieten nur begrenzte Hilfe für ein effizientes Medikamentenmanagement. Die aktuellen Interaktionsprüfungen sind nicht praxistauglich. Sortierfunktionen nach therapeutischen Gruppen und Doubletten-Elimination fehlen.

Die Einführung von therapeutischen Gruppen in den PIS ist ebenso zwingend (zur schnellen Orientierung = Effizienz) wie die konsequente Doubletten-Elimination auf Wirkstoffebene (Qualität).

- Clinical Decision Support: Längst gibt es Tools, die Hinweise geben, falls ein Medikament keine Indikation hat (fehlende Diagnose/Evidenz), eine spezielle Laborwertkontrolle überfällig ist (Management chronischer Krankheiten oder Nebenwirkungsmonitoring potentiell leberschädigender Substanzen etc.), eine erste Medikamentenkombination durch eine zweite, nebenwirkungsärmere substituierbar ist usw.

Die Anbindung von Wissensdatenbanken ist voranzutreiben und sinnvolle Tools im Bereich CDS sind einzubinden. Seitens der Fachgesellschaften sind entspre-

Heinz Bhend

info[at]dr-bhend.ch chende Ziele zu definieren und die nötigen Mittel zur Verfügung zu stellen.

- Minimale Codierung: Aktuell wird in den Spitälern v.a. nach ICD-10 codiert. Bei Austrittsberichten wird häufig dieser Code mitgeliefert und stünde für die Weiterverarbeitung in den Praxen zur Verfügung. Für Hausärzte ist ICD-10 ein No-Go. Die Nutzung von ICPC-2 ist gering, v.a. da kein Mehrwert daraus resultiert.

Die Nutzung von ICPC-2 in den (Hausarzt-)Praxen ist, auch ausserhalb von Forschungsprojekten, zu fördern. Die PIS müssen inskünftig das Mapping ICD-10 auf ICPC anbieten.

- Klinische Standards: Es herrscht ein Chaos in den PIS, wie einzelne Parameter zu definieren sind und somit als Schweizer Standard zu gelten haben. Diese Definitionsmacht haben nur die Fachgesellschaften. Aktuell wird z.B. diskutiert, nach welcher Formel die GFR berechnet werden soll und wie der Nikotinkonsum elektronisch abgebildet werden soll.

Die Fachgesellschaften (v.a. SGAIM!) definieren fachspezifische Standards für klinische Daten. Für diese Daten sind zeitnahe Standards zu definieren, welche praxistauglich implementierbar sind.

\section{Ausblick}

Nun braucht es Taten - Worte (v.a. gedruckte) sind es mehr als genug! Die angesprochenen Instanzen müssen eHealth auf ihrer Prioritätenliste nach oben schieben. Die Anekdote (Szenario links unten) ist nicht unrealistisch. Falls eine Mehrheit der niedergelassenen Ärzteschaft sich nicht für das EPD motivieren lässt, werden die Spitäler wohl kaum länger Ressourcen in das EPD investieren. Alle Vorleistungen wären dann mehr oder weniger vergeblich gewesen.

Konkret braucht es nun Projekte, welche primär einen praxisinternen Mehrwert generieren. Dazu muss die Praxisinformatik, v.a.im Bereich der IT-Unterstützung der klinischen Dokumentation, mit der gleichen Priorität weiterentwickelt werden wie aktuell das EPD.

\section{Unsere Wünsche}

Wir wünschen der FMH-Crew rund um Yvonne Gilli und Reinhold Sojer alles Gute, um die komplexe und für viele etwas undurchsichtige IT-Thematik anzugehen. Wenn es gelingt, Kolleginnen und Kollegen zu motivieren, ihre Erfahrungen einzubringen, die Wünsche und Forderungen zu bündeln und mit den Softwarefirmen den Dialog aufzunehmen, wird die nächste Generation der Praxisinformationssysteme der klinischen Tätigkeit echte Unterstützung bringen. Das Potential ist riesig. 